\title{
Traumatischer Irisverlust: Implantation einer künstlichen Iris zur Wiederherstellung von Funktion und Ästhetik
}

\author{
Christian S. Mayer · Isabella D. Baur · Julia Storr · Ramin Khoramnia
}

Eingegangen: 28. Juni 2021 / Angenommen: 2. September 2021 / Online publiziert: 6. Oktober 2021

(c) Der/die Autor(en) 2021

\begin{abstract}
Zusammenfassung
Ziel Wir berichten über den Fall eines Patienten mit traumatischem Irisdefekt, der sich 4 Monate nach dem initialen Trauma im Rahmen eines Fahrradunfalls einer chirurgischen Irisrekonstruktion mit einer CUSTOMFLEX ArtificialIris (AI, HumanOptics AG, Erlangen, Deutschland) unterzog.

Beobachtungen Bei der Erstvorstellung zeigte sich eine gedeckte Bulbusruptur, die notfallmäßig mittels Vorderkammerspülung, Vitrektomie, Skleranaht und $\mathrm{C}_{3} \mathrm{~F}_{8}$ Gastamponade versorgt wurde. Nach extern durchgeführter sekundärer IOL-Implantation klagte der Patient über eine erhöhte Blendempfindlichkeit und Visusminderung am rechten Auge. Der bestkorrigierte Fernvisus betrug 1,4 logMAR für das rechte Auge und 0,10 logMAR für das linke Auge. Die klinische Untersuchung zeigte einen großen Irisdefekt mit Restirisgewebe von 4 bis $8 \mathrm{Uhr}$. Beide Augen waren pseudophak, der fundoskopische Befund war unauffällig. Der Patient unterzog sich einer AI-Implantation v. a. zur Linderung seiner Blendempfindlichkeit am rechten Auge. Die Sehschärfe stieg auf 0,30 logMAR an. Die subjektive Blendempfindlichkeit verbesserte sich deutlich. Die Kontrastempfindlichkeit, gemessen mit einer Pelli-Robson-Tafel, stieg von 0,15 logarithmischen Einheiten präoperativ auf 1,05 logarithmische Einheiten postoperativ.
\end{abstract}

Die Autoren Christian S. Mayer und Isabella D. Baur teilen sich die Erstautorenschaft.

PD Dr. C. S. Mayer $(\varangle) \cdot$ I. D. Baur · R. Khoramnia Augenklinik, Universitätsklinikum Heidelberg, Im Neuenheimer Feld 400, 69120 Heidelberg, Deutschland Christian.Mayer@med.uni-heidelberg.de

\section{J. Storr}

Klinik und Poliklinik für Augenheilkunde, Klinikum rechts der Isar, München, Deutschland
Schlussfolgerungen Wir beobachteten nach chirurgischer Irisrekonstruktion mit einer künstlichen Iris ein sehr gutes ästhetisches und funktionelles Ergebnis mit hoher Patientenzufriedenheit. Dieser Fall demonstriert, wie die Reduktion der Blendempfindlichkeit gleichzeitig auch zu einer deutlichen Verbesserung der Funktion mit Anstieg von Sehschärfe und Kontrastsehen führen kann.

Schlüsselwörter Irisdefekt · Trauma · Perforierende Augenverletzungen · Irisimplantat · Chirurgische Irisrekonstruktion

Traumatic iris defect: implantation of an artificial iris to restore function and aesthetics

\section{Summary}

Purpose To present the case of a patient with a traumatic iris defect who underwent surgical iris reconstruction with a CUSTOMFLEX ArtificialIris (AI, HumanOptics AG, Erlangen, Germany) 4 months after the initial trauma, which took place in the context of a bicycle accident.

Observation At the initial presentation, we observed an open eyeball injury, that was immediately surgically treated including vitrectomy, anterior chamber irrigation and suturing of the sclera and $\mathrm{C}_{3} \mathrm{~F}_{8}$ gas endotamponade. After secondary IOL implantation at another clinic, the patient complained about increased glare sensitivity and decreased visual acuity in the right eye. Corrected distance visual acuity was $1.4 \operatorname{logMAR}$ for the right eye and $0.10 \log$ MAR for the left eye. Slit lamp examination revealed a large iris defect with residual iris tissue from 4 o'clock to 8 o'clock. Both eyes were pseudophakic and funduscopy was unremarkable. The patient underwent sulcus fixated AI implantation of the right eye. Visual acuity improved to $0.30 \log$ MAR. Subjective glare 
sensitivity improved considerably. Contrast sensitivity measured with the Pelli-Robson chart improved from $0.15 \log$ units preoperatively to $1.05 \log$ units postoperatively.

Conclusion We observed a very good aesthetic and functional result after surgical iris reconstruction with an artificial iris. This case demonstrates how correction of glare sensitivity can lead to a significant increase in visual acuity and in contrast sensitivity.

Keywords Iris defect - Trauma - Open globe injuries · Iris implant · Surgical iris reconstruction

Irisdefekte können neben einer kosmetischen Beeinträchtigung auch eine vermehrte Blendung und reduzierte Sehschärfe hervorrufen [1]. Zu den konservativen Therapieoptionen zählen das Tragen einer Sonnenbrille oder von bedruckten Iriskontaktlinsen. Während eine Sonnenbrille nur eine vorübergehende Linderung der Blendung bewirken kann, können Irisprintkontaktlinsen ein gutes ästhetisches Ergebnis erreichen und die Blendung effektiv reduzieren [2]. Ein sichtbarer Seitenunterschied zwischen dem gesunden Auge und dem mit der Irisprintkontaktlinse versorgten Auge kann jedoch zur Unzufriedenheit durch den auffälligen ästhetischen Eindruck führen. Zudem sind die richtige Handhabung der Kontaktlinsen und ihre Pflege für manche Patienten schwierig. Das Tragen von Kontaktlinsen birgt auch ein Infektionsrisiko, und die Toleranz von Kontaktlinsen kann bei irregulärer Hornhaut oder trockenem Auge reduziert sein. Die Kontaktlinsen müssen zudem regelmäßig ersetzt werden und generieren so fortlaufend Kosten. Der Wunsch nach einer einmaligen, dauerhaften Lösung durch die chirurgische Korrektur des Irisdefektes ist daher nachvollziehbar.

\section{Falldarstellung}

\section{Anamnese}

Ein 84-jähriger Patient stellte sich in unserer Sprechstunde mit einem traumatischen Irisdefekt vor, den er sich bei einem Fahrradunfall 4 Monate zuvor zugezogen hatte.

Bei der Erstvorstellung nach dem Unfall zeigte sich eine gedeckte Bulbusruptur mit Ruptur der Sklera von 10 bis 2 Uhr sowie Iris- und Glaskörperprolaps und traumatischem Linsenverlust sowie traumatischem Irisdefekt. Die Verletzung wurde notfallmäßig mittels Vorderkammerspülung, Vitrektomie und Skleranaht versorgt. Zudem wurde ein Netzhautforamen bei 12 Uhr mittel Kryopexie und $\mathrm{C}_{3} \mathrm{~F}_{8}$-Luft-Gas-Gemisch behandelt. Das Auge wurde im Rahmen der Primärversorgung zunächst aphak belassen. Extern erfolgte dann eine sekundäre Linsenimplantation, nach der sich der Patient erneut in unserer Sprechstunde vorstellte. Der Patient beklagte zu diesem Zeitpunkt eine starke Blendempfindlichkeit und Visusminderung am betroffenen rechten Auge.

\section{Befund}

Der bestkorrigierte Visus am betroffenen rechten Auge lag bei 1,4 logMAR mit einer Refraktion von -0,75/ plan/-, am linken Auge zeigte sich ein bestkorrigierter Visus von 0,10 logMAR. In der Spaltlampenuntersuchung zeigte sich am rechten Auge ein ausgeprägter Irisdefekt mit Restirisgewebe im Bereich von 4 bis 8 Uhr bei klarer Hornhaut und Pseudophakie. Die Abb. 1a, b zeigt den präoperativen Befund mit Irisdefekt am rechten Auge. Der Fundus war unauffällig. Am linken Auge zeigte sich eine reizfreie Pseudophakie bei fundoskopischem Normalbefund. Der in-
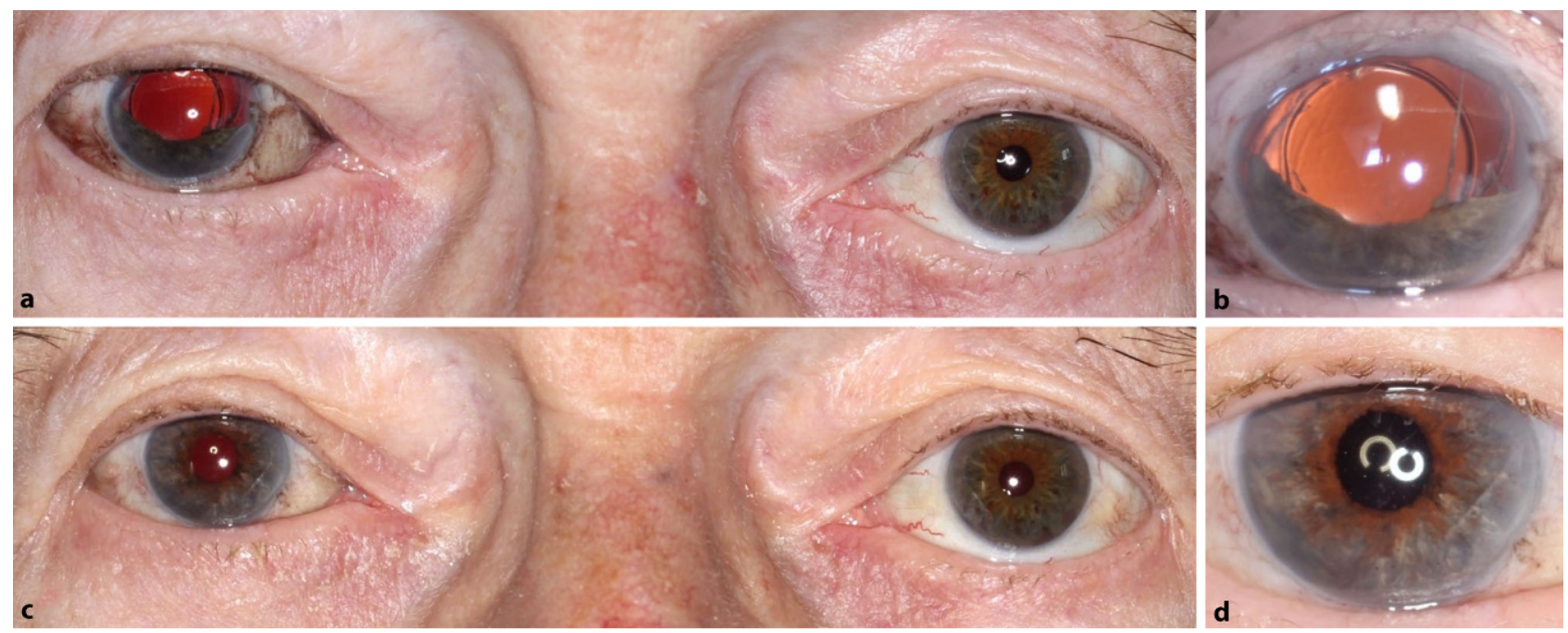

Abb. 1 a Übersichtsaufnahme des präoperativen Befundes. b Nahaufnahme des präoperativen Befundes. Die Intraokularlinse sowie der ausgeprägte Irisdefekt am rechten Auge sind deutlich zu erkennen. c Übersichtsfoto des postoperativen

Befundes. d Nahaufnahme des postoperativen Befundes am rechten Auge. Die Al ist gut zentriert und kaum vom natürlichen Irisgewebe abgrenzbar 

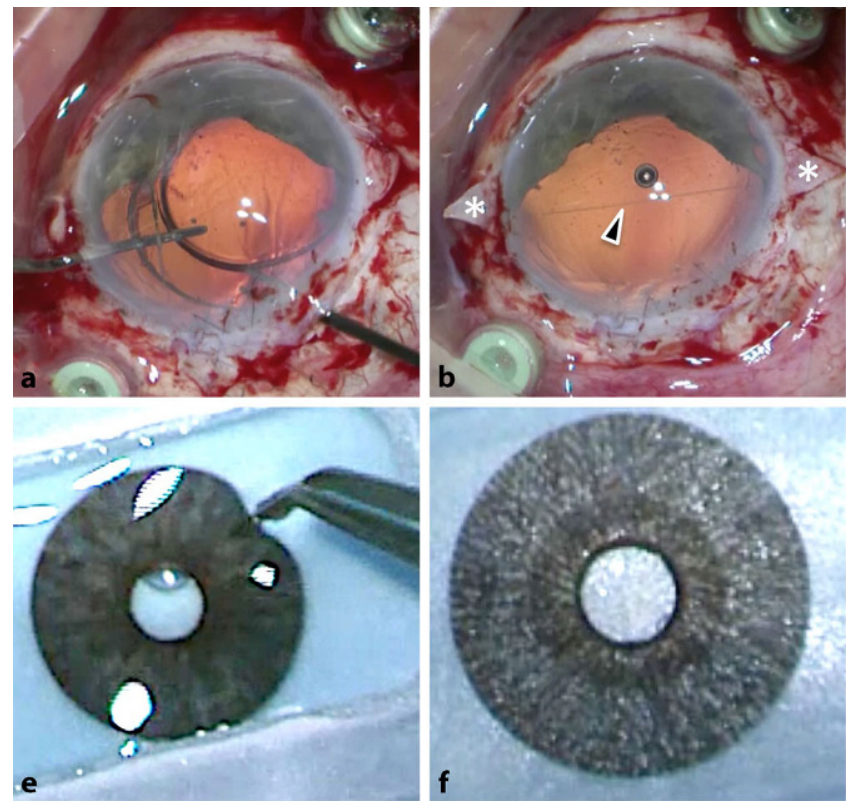

Abb. 2 a Fassen der subluxierten IOL und Explantation durch einen sklerokornealen Tunnel unter Vitrektomiebereitschaft. b Präparation der Skleradeckel (Sterne) und Vorlegung der quer durch den Sulcus ciliaris führenden Fixationsnaht (Pfeil). Der Faden wird mit einem Häkchen herausgeholt, durchtrennt, und die Enden werden an den jeweiligen IOL-Haptiken angenäht. c Implantation der sulcusfixierten und starren PMMA-Intraokularlinse. d Ausrichtung und Verknoten der IOL-Nähte unter den Skleradeckeln in der 3- und 9-UhrPosition. e Entnahme aus der Verpackung und Vorbereitung

traokulare Druck am rechten Auge lag mit $19 \mathrm{~mm} \mathrm{Hg}$ im Normbereich.

Der Patient wurde gebeten, die subjektive Blendung sowie die ästhetische Beeinträchtigung auf einer Skala von 1 bis $10 \mathrm{zu}$ bewerten, wobei 1 jeweils für „keine Beeinträchtigung“ und 10 für „starke Beeinträchtigung“ steht. Der Patient gab für die Beeinträchtigung durch Blendung den Wert 9 und für die ästhetische Beeinträchtigung den Wert 3 an.

Die Kontrastempfindlichkeit wurde mit einer Pelli-Robson-Tafel gemessen. Am rechten Auge erreichte der Patient einen Wert von 0,15 logarithmischen Einheiten.

\section{Therapie und Verlauf}

Um das subjektive Blendungsempfinden zu reduzieren, schlugen wir dem Patienten die Implantation einer künstlichen Iris (CUSTOMFLEX ArtificialIris, AI, HumanOptics AG, Erlangen, Germany) am rechten Auge vor. Nach ausführlicher Beratung und Aufklärung über konservative und alternative Therapieoptionen und mögliche Komplikationen sowie entsprechender Bedenkzeit entschied sich der Patient für den Eingriff.

Präoperativ wurde eine optische Biometrie mit dem IOL Master 500 (Carl Zeiss, Jena, Deutschland) durchgeführt. Die erforderliche Intraokularlinse wur-
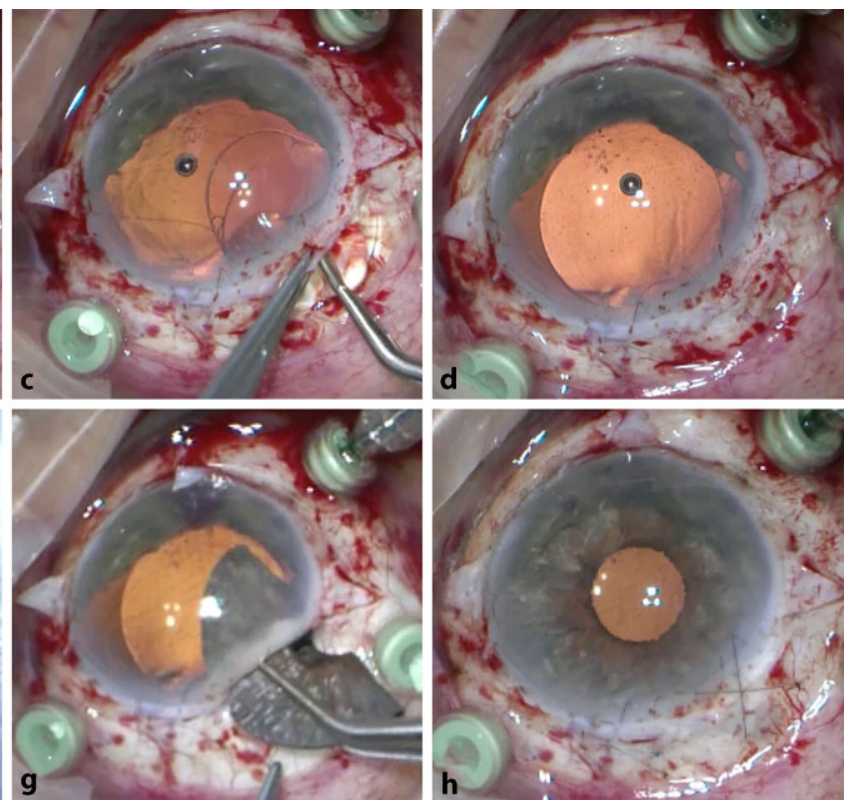

des flexiblen Silikonimplantates. Die Artificial/ris wird extraokular auf die richtige Größe mit einem Einmaltrepan auf den Sulcusdurchmesser zurechtgestanzt. f Vorderseite der ArtificialIris vor der Implantation. Die hier nicht sichtbare Rückseite ist schwarz und glatt. $\mathbf{g}$ Das gefaltete Irisimplantat wird über den sklerokornealen Tunnel implantiert und $\mathbf{h}$ ebenfalls mit 2 Nähten - diesmal aber in der 6-Uhr- und 12-Uhr-Position, um ein Verkippen auf der IOL zu verhindern - im Sulcus fixiert. $\mathbf{h}$ Endergebnis mit zentrierter IOL und vernähtem sklerokornealem Tunnel und Skleradeckeln vor Verschluss der Bindehaut

de mithilfe der Haigis-Formel ohne Korrekturfaktor berechnet, wobei eine emmetrope Zielrefraktion angestrebt wurde. Wir konnten in einer anderen Arbeit zeigen, dass bei dem gewählten operativen Vorgehen kein Korrekturfaktor erforderlich ist und die Standardformeln zur IOL-Berechnung angewendet werden können [3].

Die Operation wurde in Allgemeinanästhesie durchgeführt. Die Abb. 2 zeigt die einzelnen Schritte des Eingriffs. Bei nicht ausreichender Stabilität der bestehenden sklerafixierten IOL und drohender (Sub-)Luxation der AI vor der IOL wurde diese explantiert (Abb. 2a) und durch eine neue, nahtfixierte IOL in der 3-und 9-Uhr-Position ersetzt (Abb. 2b-d). Es wurde eine zuvor individuell angefertigte AI mit eingearbeitetem Meshgewebe ausgesucht, die auf einen Durchmesser von 11,5 mm zugestanzt wurde. Anschließend wurde sie (Abb. 2e, f) anterior im Sulcus ciliaris bei 6 und $12 \mathrm{Uhr}$ nahtfixiert implantiert (Abb. 2g, h). Diese Art der Fixierung wurde gewählt, um einer axialen Verkippung der Implantate vorzubeugen [1]. Sowohl zur Fixierung der IOL als auch der AI wurden jeweils 2 Sklerataschen präpariert, und zum Einbringen der Implantate in das Auge wurde ein sklerokornealer Tunnel angelegt. Die Ankerfäden für die IOL und AI wurden mit doppeltarmierten 10-0 Prolene-Fäden bei 3 und $9 \mathrm{~h}$ bzw. 6 und $12 \mathrm{~h}$ vorgelegt. Die Implantate wurden dann über den sklerokornealen 
Tunnel implantiert, und die Prolene-Fäden mit den Schlittennadeln angezogen und in den Sklerataschen verknotet. Der sklerokorneale Tunnel wurde mit drei 10-0 Nylon-Kreuzstichnähten verschlossen.

Die Abb. 1c, d zeigt das postoperative Ergebnis. Die $\mathrm{AI}$ ist gut zentriert, der Übergang zwischen dem Implantat und dem natürlichen Irisgewebe ist auch in der Nahaufnahme (Abb. 1d) kaum zu erkennen. Drei Monate postoperativ konnten wir einen bestkorrigierten Visus von 0,30 logMAR mit einer Refraktion von $+2,75 /-3,25 / 90^{\circ}$ feststellen. Der intraokulare Druck lag mit $18 \mathrm{~mm} \mathrm{Hg}$ weiterhin im Normbereich.

Der Patient bewertete die subjektive Blendung jetzt mit 4 und die ästhetische Beeinträchtigung mit 1 jeweils auf einer Skala von 1 bis 10 . Der Patient wurde auch gebeten, das Gesamtergebnis auf einer Skala von 1 bis $10 \mathrm{zu}$ bewerten, wobei 1 für geringe und 10 für maximale Zufriedenheit steht. Hier gab der $\mathrm{Pa}$ tient einen Wert von 9 an.

Die Kontrastempfindlichkeit, gemessen mit einer Pelli-Robson-Tafel, stieg auf 1,05 logarithmische Einheiten an.

\section{Diskussion}

Wir konnten bei unserem Patienten ein sehr gutes ästhetisches Ergebnis sowie eine effektive Reduktion der Blendung erreichen. Der bestkorrigierte Visus stieg deutlich an, und der Patient war sehr zufrieden mit dem Ergebnis.

In diesem Fall wurden die Intraokularlinse und ArtificialIris jeweils einzeln nahtfixiert implantiert. Alternativ kann die IOL auch auf die AI aufgenäht und beide Implantate können kombiniert eingenäht werden $[1,4,5]$. An der optischen Bank konnte gezeigt werden, dass auch das Zusammennähen beider Implantate die optische Qualität nur unwesentlich beeinflusst [6]. Die Implantation kann - je nach Ausgangssituation - auch in den Kapselsack oder Sulcus ciliaris ohne Nahtfixierung erfolgen [1]. So kann die chirurgische Vorgehensweise individuell angepasst werden. Weder bei der kapselsackfixierten noch bei der nahtfixierten Implantation einer Intraokularlinse zusammen mit der AI muss von den Standardformeln zur IOL-Berechnung abgewichen werden, es ist kein Korrekturfaktor erforderlich [3].

Da die CUSTOMFLEX-AI individuell anhand eines Fotos von Hand bemalt wird, um sie dem restlichen Irisgewebe bzw. dem Partnerauge möglichst genau anzupassen, können damit, wie auch in diesem Fall, sehr gute ästhetische Ergebnisse erzielt werden [1, 7-12]. Zur Anfertigung des Implantates muss im Vorfeld ein farbtreues Foto angefertigt werden, nach dessen Vorlage die AI angefertigt wird. Aufgrund der Individualisierung des Implantates dauert die Herstellung mehrere Wochen.

Die AI kann durch die Verkleinerung der Pupille Blendung effektiv reduzieren und das Kontrastsehen signifikant verbessern $[9,11,13]$. Auch eine beidseiti- ge Implantation bei Patienten mit bilateralen Irisdefekten ist möglich, in diesen Fällen können ebenfalls gute funktionelle und ästhetische Ergebnisse erzielt werden [14].

Ein Visusanstieg ist nicht das primäre Ziel einer AIImplantation und kann abhängig davon, welche Begleitverletzungen vorliegen, nicht in allen Fällen erwartet werden. Trotzdem wurde über eine Verbesserung des Visus nach der Implantation schon mehrfach berichtet und kann oftmals auch durch eine im gleichen Eingriff durchgeführte sekundäre IOL-Implantation oder chirurgische Therapie einer (traumatischen) Katarakt erklärt werden $[1,10,14,15]$. Im hier beschriebenen Fall lag präoperativ weder eine Aphakie noch eine traumatische Katarakt vor, sodass der Visusanstieg trotz eines vermutlich operativ induzierten Astigmatismus am ehesten durch die Reduktion der Blendung zu erklären ist.

Neben den Chancen, mit denen eine AI-Implantation verbunden ist, sollten auch mögliche Risiken vor dem Eingriff mit dem Patienten diskutiert werden. $\mathrm{Zu}$ den beschriebenen Komplikationen nach AI-Implantation zählen u.a. die Dislokation des Implantates, Entwicklung oder Dekompensation eines Glaukoms, eine Hornhautdekompensation und das Auftreten eines Makulaödems [10, 16]. Das Risiko für verschiedene Komplikationen hängt von der Ausgangssituation bzw. den Komorbiditäten des jeweiligen Auges ab. So ist bei bereits präoperativ reduzierter Endothelzellzahl das Risiko einer Hornhautdekompensation erhöht und bei Glaukompatienten ein postoperativer Augendruckanstieg häufiger zu erwarten. Eine weitere Komplikation in Zusammenhang mit der AI-Implantation ist das sog. „residual iris retraction syndrome“ (RITS) [17]. Aus bislang ungeklärter Ursache vergrößert sich beim RITS die natürliche Pupillenöffnung des verbliebenen natürlichen Irisgewebes Monate bis Jahre nach der Implantation zunehmend. Die Veränderung kann zu einem Winkelblock- oder Pigmentdispersionsglaukom führen und wurde insbesondere bei Patienten mit sulcusfixierter AI, nicht jedoch nach kapselsackfixierter oder nahtfixierter Implantationstechnik beschrieben [17]. Auch eine Änderung der Farbe des verbliebenen natürlichen Irisgewebes wurde in einigen Fällen beobachtet. Das kosmetische Ergebnis kann dadurch negativ beeinflusst werden [13].

Das Ophtec Iris-Implantat (Ophtec GmbH, Groningen, Niederlande) ist eine mögliche Alternative zum hier verwendeten Implantat [18]. Es steht in über 120 Farbvariationen zur Auswahl und ist mit oder ohne Optik verfügbar. Das Implantat kann ebenfalls in den Kapselsack implantiert oder in den Sulcus eingenäht werden.

Eine weitere Option zur Korrektur der Aniridie sind die Aniridieimplantate der Firma Morcher (Stuttgart, Deutschland), z. B. das Implantat Typ 68 mit integrierter Optik. Dieses Implantat ist nicht faltbar und nur in schwarzer Farbe erhältlich. 
Wir verwendeten die CUSTOMFLEX AI der Firma HumanOptics, da das Implantat individuell für den jeweiligen Patienten angefertigt wird und mit einer Vielzahl an Intraokularlinsen kombinierbar ist. Somit ermöglicht es eine speziell auf den Patienten zugeschnittene Therapie.

Wir beobachteten ein sehr gutes ästhetisches Ergebnis sowie eine deutliche Verbesserung der Funktion am rechten Auge. Dieser Fall demonstriert, wie die Implantation der AI nicht nur zu einer Verbesserung des ästhetischen Eindrucks, sondern auch zur Korrektur der Blendempfindlichkeit mit einem deutlichen Anstieg der Sehschärfe und der Kontrastsensitivität führen kann.

Funding Open Access funding enabled and organized by Projekt DEAL.

\section{Einhaltung ethischer Richtlinien}

Interessenkonflikt C.S. Mayer erhielt Reisestipendien und Vortragshonorare von HumanOptics. I.D. Baur, J. Storr und R. Khoramnia geben an, dass kein Interessenkonflikt besteht.

Ethische Standards Alle angewandten Verfahren entsprachen den ethischen Standards des zuständigen Komitees für Menschenversuche (institutionell und national) und der Deklaration von Helsinki von 1975 in der 2008 überarbeiteten Fassung. Eine schriftliche Einwilligung des Patienten wurde eingeholt.

Open Access Dieser Artikel wird unter der Creative Commons Namensnennung 4.0 International Lizenz veröffentlicht, welche die Nutzung, Vervielfältigung, Bearbeitung, Verbreitung und Wiedergabe in jeglichem Medium und Format erlaubt, sofern Sie den/die ursprünglichen Autor(en) und die Quelle ordnungsgemäß nennen, einen Link zur Creative Commons Lizenz beifügen und angeben, ob Änderungen vorgenommen wurden.

Die in diesem Artikel enthaltenen Bilder und sonstiges Drittmaterial unterliegen ebenfalls der genannten Creative Commons Lizenz, sofern sich aus der Abbildungslegende nichts anderes ergibt. Sofern das betreffende Material nicht unter der genannten Creative Commons Lizenz steht und die betreffende Handlung nicht nach gesetzlichen Vorschriften erlaubt ist, ist für die oben aufgeführten Weiterverwendungen des Materials die Einwilligung des jeweiligen Rechteinhabers einzuholen.

Weitere Details zur Lizenz entnehmen Sie bitte der Lizenzinformation auf http://creativecommons.org/licenses/by/4. 0/deed.de.

\section{Literatur}

1. Mayer C, Tandogan T, HoffmannAE, Khoramnia R. Artificial iris implantation in various iris defects and lens conditions. J Cataract RefractSurg. 2017;43(6):724-31.

2. Burger DS, London R. Soft opaque contactlenses in binocular vision problems. JAm Optom Assoc. 1993;64:176.

3. Mayer C, Khoramnia R. "Double prosthesis implantation": biometry and refractive outcomes in combined in- traocular lens and artificial iris surgery. Clin Ophthalmol. 2021;15:799-805.

4. Mayer C, Baur ID, Storr J, Khoramnia R. Complete anterior segment reconstruction: corneal transplantation and implantation of an iris prosthesis and IOL in a single surgery. Eur J Ophthalmol. 2021; https://doi.org/10.1177/ 1120672121991052.

5. Szurman P, Jaissle G. Artificial iris. Ophthalmologe. 2011;108(8):720.

6. Mayer C, Son H-S, Labuz G, Yildirim TM, Auffarth GU, Khoramnia R. In vitro optical quality assessment of a monofocal IOL sutured to an artificial iris. J Cataract Refract Surg. 2020;46(8):1184-8.

7. Yildirim TM, Khoramnia R, Masyk M, Son H-S, Auffarth GU, Mayer CS. Aesthetics of iris reconstruction with a custom-made artificial iris prosthesis. PLoS ONE. 2020;15(8):e237616.

8. Mayer CS, BaurID, Storr J, Khoramnia R. Traumatic aniridia: conservative or surgical therapeutic approach? Ophthalmologe.2021; https://doi.org/10.1007/s00347-021-013678.

9. Mayer CS, Reznicek L, Hoffmann AE. Pupillary reconstruction and outcome after artificial iris implantation. Ophthalmology. 2016;123(5):1011-8.

10. Spitzer MS, Nessmann A, Wagner J, Yoeruek E, BartzSchmidt KU, Szurman P, et al. Customized humanoptics silicone iris prosthesis in eyes with posttraumatic iris loss: outcomes and complications. Acta Ophthalmol. 2016;94(3):301-6.

11. Spitzer MS, Yoeruek E, Leitritz MA, Szurman P, BartzSchmidt KU. A new technique for treating posttraumatic aniridia with aphakia: first results of haptic fixation of a foldable intraocular lens on a foldable and custom-tailored iris prosthesis. Arch Ophthalmol. 2012;130(6):771-5.

12. Baur ID, Mayer CS, Storr J, Khoramnia R. Artificial iris implantation in a patient with iatrogenic iris defect following cataract surgery. Klin Monbl Augenheilkd. 2021;238(8):875-7.

13. Rickmann A, Szurman P, Januschowski K, Waizel M, Spitzer MS, Boden KT, et al. Long-term results after artificial iris implantation in patients with aniridia. Graefes Arch Clin Exp Ophthalmol.2016;254(7):1419-24.

14. Mayer CS, Baur ID, Storr J, Khoramnia R. Bilateral artificial iris implantation in patients with bilateral iris defects. Am J Ophthalmol Case Rep. 2021;22:101108.https://doi.org/ 10. 1016/j.ajoc.2021.101108.

15. Mayer CS, Hoffmann AM, Prahs P, ReznicekL, Khoramnia R. Functional outcomes after combined iris and intraocular lens implantation in various iris and lens defects. BMC Ophthalmol.2020;20(1):370.

16. Mayer CS, Laubichler AE, Khoramnia R, Tandogan T, Prahs P, Zapp D, et al. Challenges and complication management in novel artificial iris implantation. JOphthalmol. 2018;2018:3262068. https://doi.org/10.1155/2018/ 3262068.

17. Mayer CS, Laubichler AE, Masyk M, Prahs P, Zapp D, Khoramnia R. Residual iris retraction syndrome after artificial iris implantation. Am J Ophthalmol. 2019;199:159-66.

18. Price MO, Price FW Jr, Chang DF, Kelley K, Olson MD, Miller KM. Ophtec iris reconstruction lens United States clinicaltrialphaseI.Ophthalmology.2004;111(10):1847-52.

Hinweis des Verlags Der Verlag bleibt in Hinblick auf geografische Zuordnungen und Gebietsbezeichnungen in veröffentlichten Karten und Institutsadressen neutral. 